\title{
Symptomatic Tophaceous Gout in the Bilateral Patellae
}

\section{Dae Geun Kim', Gil Yeong Ahn²}

'Department of Orthopedic Surgery, Soonchunhyang University Gumi Hospital, Gumi; ${ }^{2}$ Department of Orthopedic Surgery, Pohang St. Mary's Hospital, Pohang, Korea

Tophaceous gout is an inflammatory arthropathy caused by hyperuricemia. Gout shows typically episodic acute and chronic pain with arthritis due to synovitis induced by deposition of monosodium urate crystals. Tophus which is deposits of crystals could be formed around mainly peripheral joints such as the first metatarsophalangeal joint but might be presented in any other joints. Even though gout of unilateral patella has been reported severally, tophaceous gout of bilateral patellae has been rarely seen. We present a case of gout of bilateral patellae with literature review.

Keywords: Patella; Gout; Tophi

\section{INTRODUCTION}

Gout is an inflammatory arthritis caused by an inflammatory reaction to monosodium urate crystals in the joint [1]. Tophi which are monosodium urate crystal deposits in the synovium are caused by poor solubility of the synovial fluid and the lower temperatures [2]. Therefore, tophi usually involve the peripheral joints such as the first metatarsophalangeal joint, ankle joint, and so on [3]. Tophi can present around knee joint about 7\% among the patients who has tophaceous gout [4]. While solitary gout tophus of unilateral patella has been reported, tophaceous gout of bilateral patellae is rare.

Herein we present a case of tophaceous gout on bilateral patellae and review of the literature.

\section{CASE REPORT}

A 49-year-old gentleman presented to outpatient department of orthopedic surgery, Pohang St. Mary's Hospital with a complaint of multiple growing masses and pain involving the anterior aspect of his both knees. Two masses of right knee and one mass of left knee were palpable (Fig. 1). The masses appeared 3 years prior to his visit and the pain around the masses had gotten worse over time. The patient had been diagnosed with gout for 1 year but was only treated with naproxen just for pain control.

The physical examination revealed that there was mild tenderness around the masses, but no heating sense. The passive and active range-of-motion was normal. Laboratory test revealed that white blood cells count, erythrocyte sedimentation rate, and C-reactive protein level is normal except uric acid level is high such as $8.3 \mathrm{mg} / \mathrm{dL}$ (normal range, $4-7 \mathrm{mg} / \mathrm{dL}$ ).

Plain radiographs showed a well-defined geographic $8 \times 16-\mathrm{mm}$ sized osteolytic lesion with sclerotic border involving the superolateral part of the right patella (Fig. 2). Magnetic resonance imaging (MRI) revealed three soft tissue masses. Two masses in the right patella and one mass in the left patella were confirmed. MRI showed these masses had low signal intensity on T1-weighted sequences and inhomogeneous high signal intensity on T2-weighted images with soft tissue infiltration (Fig. 3).

The patient decided upon surgical excision of the masses due to pain and size. At surgery, midline longitudinal incision was made, then the patella and retinaculum were exposed. The masses consisted of chalky material having texture of gout tophi. The three lesions were excised with massive curettage (Fig. 4). Histologic examination of the specimen revealed numerous large tophi composed of long, refractile, needle-shaped crystals. Pain gradually
Correspondence to: Dae Geun Kim

Department of Orthopedic Surgery, Soonchunhyang University Gumi Hospital, 179 1(il)gongdan-ro, Gumi 39371, Korea

Tel: +82-54-468-9124, Fax: +82-54-463-7504, E-mail: kuroo25@schmc.ac.kr

Received: Feb. 11, 2019 / Accepted after revision: Jun. 17, 2019
(C) 2019 Soonchunhyang Medical Research Institute This is an Open Access article distributed under the terms of the Creative Commons Attribution Non-Commercial License (http://creativecommons.org/licenses/by-nc/4.0/). 

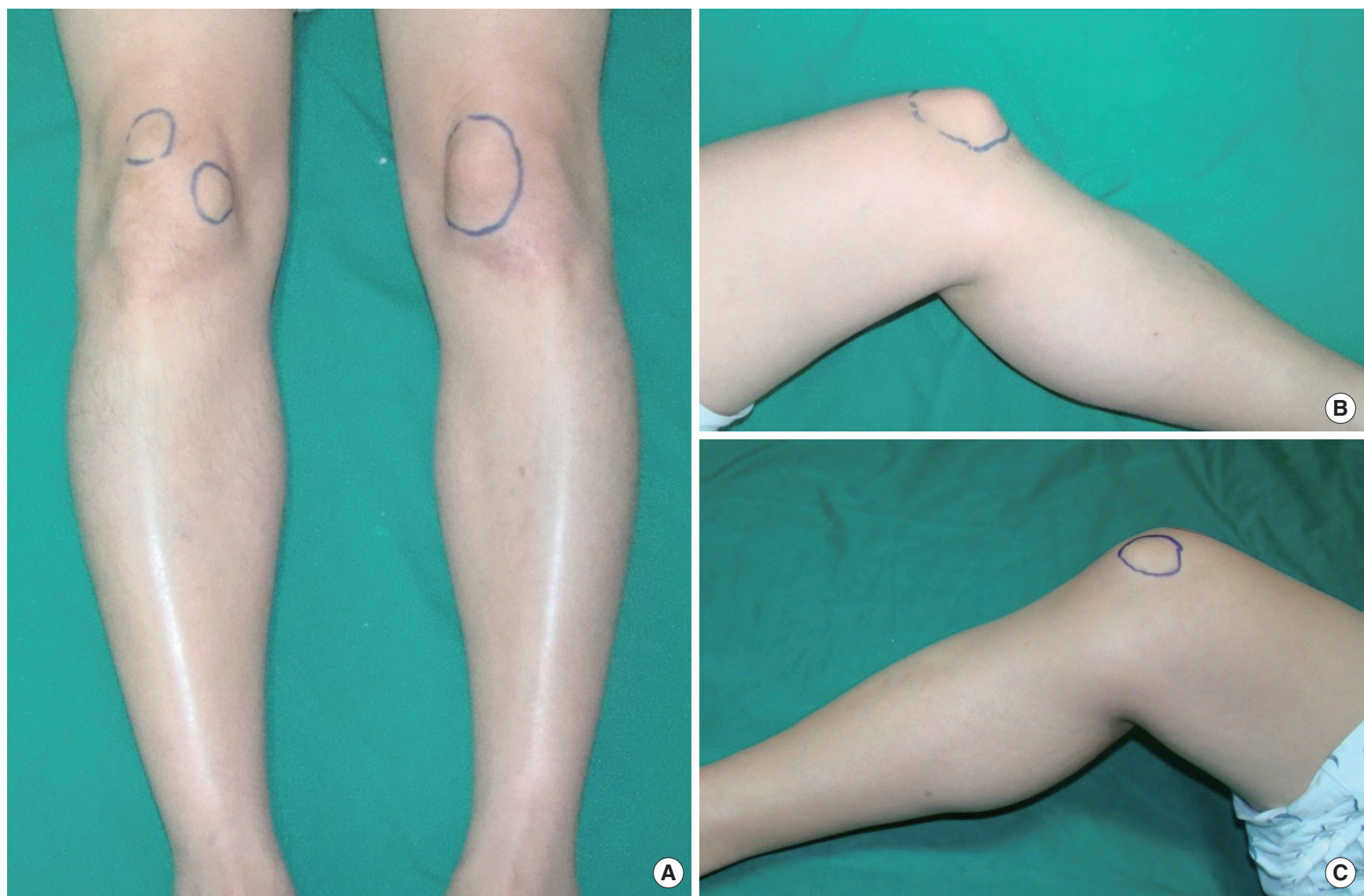

Fig. 1. (A-C) There are two masses on right knee and one mass on left knee.
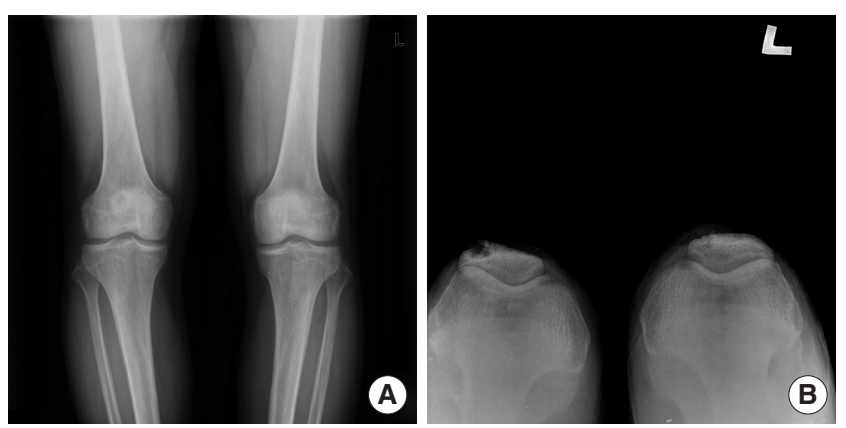

resolved after surgery and febuxostat was administrated at postoperative 2 weeks. The patient has no tophaceous gout and his serum uric acid level is well controlled such as $5.8 \mathrm{mg} / \mathrm{dL}$ at 1 -year follow-up. And written informed consent was obtained from the patient.

\section{DISCUSSION}
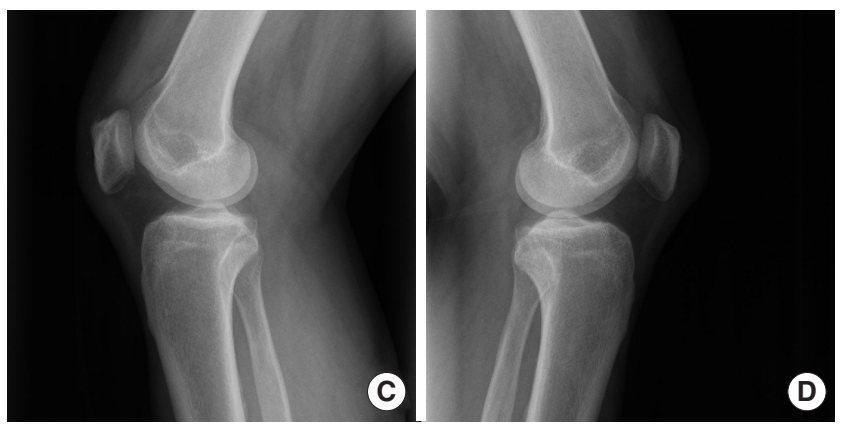

Fig. 2. (A-D) Preoperative plain radiographs demonstrate a well-defined geographic osteolytic lesion with sclerotic border involving the superolateral part of right patella.

Gout is an inflammatory arthropathy due to hyperuricemia. Hyperuricemia result in a high concentration of monosodium urate crystals, which can deposit in cartilage, bone, and joint spaces causing an inflammatory reaction [1]. A tophus results from an organization of monosodium urate crystals and inflammatory cells [2]. Any joint could be involved in gout, but the most common joint is the first metatarsophalangeal joint due to low solubility [3].

Tophaceous gout of the patella is not common. Peloquin and Graham [5] first reported gout of the patella in 1955. Although several cases with solitary tophaceous gout of the patella have been 

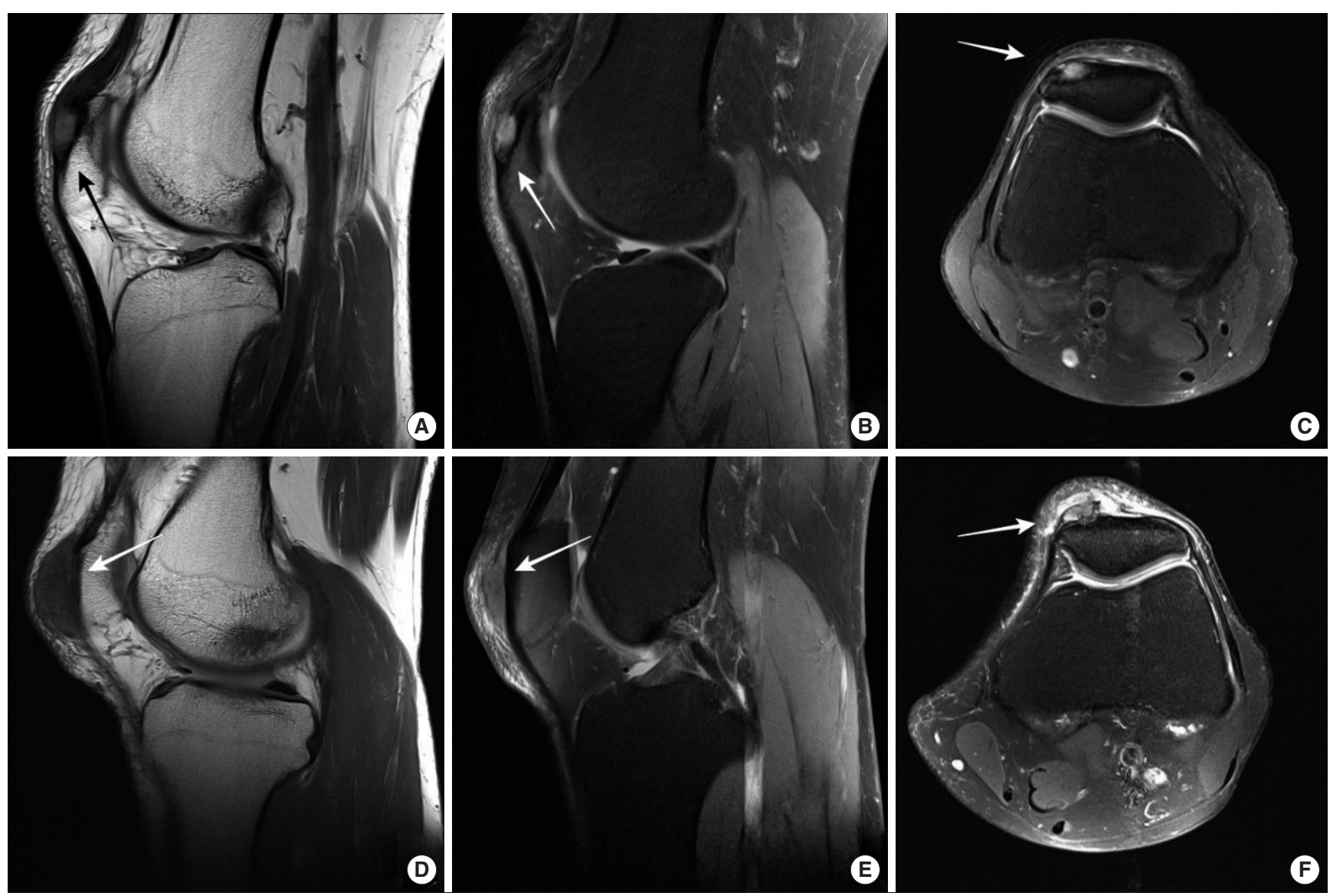

Fig. 3. Magnetic resonance imaging scan reveals three soft tissue masses. $(A-C)$ In the right patella, two masses are located on the superolateral part and the medial part (arrow). (D-F) In the left patella, a mass is located on the medial aspect of the patella (arrow). The signal intensity of the lesion shows low signal intensity on T1weighted sequences and inhomogeneous high signal intensity on T2-weighted images with soft tissue infiltration.
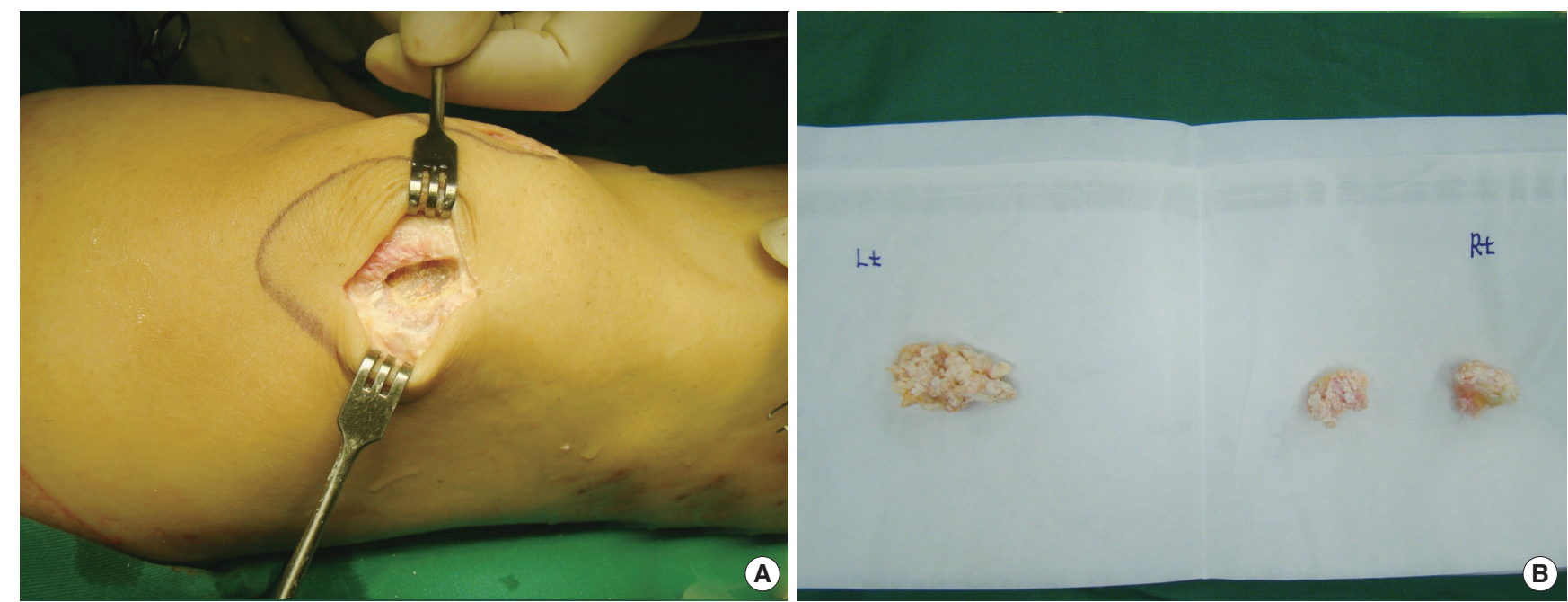

Fig. 4. (A) The masses are excised with curettage. (B) All the masses are chalky material that look alike gouty tophi.

reported ever since, gout tophi in the bilateral patella have been reported rarely [6].
Typical radiologic findings of tophaceous gout include juxta and intra-articular bony erosion with sclerotic margins and over- 
hanging edges. Bony erosion could be led to synovitis induced by crystal deposition [7]. Typically, locations of gout tophi are the superolateral aspect of the patella. In our case, the largest mass was located in the superolateral side of the patella and showed osteolytic lesion in the plain radiographs. Because radiographic findings in acute gouty arthritis are apt to be minimal or absent, consisting of periarticular soft tissue swelling, smaller mass didn't show osteolytic lesion in the plain radiographs.

MRI characteristics of tophi include low-intermediate signal intensities on T1-weighted images, with heterogeneous signal intensities on T2-weighted images [8]. Our patient's MRI shows high signal intensity on T2-weighted images because of inflammation.

The differential diagnosis of osteolytic lesion in the patella includes infection, metabolic diseases, degenerative conditions, tumors, and so on. The most common reported causes of osteolytic lesion in the patella in patients younger than 40 years of age were chondroblastoma and giant cell tumor, while metastatic tumor and gout were the most common for patients over 40 years old [9]. In our case, the patient is over 40 years of age and he has the history of gouty arthritis. Therefore, we could suspect the masses in the bilateral patellae would be gout tophi.

In conclusion, we have to keep in mind that a mass of any joint could be gouty tophus in the gout patients even though topha- ceous gout in bilateral patellae are rare. Gout is a progressive reactive arthritis, so that excision of tophus is not enough treatment of gout. Tophaceous gout could be prevented by proper diet and medication such as xanthine oxidase inhibitors, probenecid, or pegloticase which is an enzyme that metabolizes uric acid into a harmless chemical.

\section{REFERENCES}

1. Weselman KO, Agudelo CA. Gout basics. Bull Rheum Dis 2001;50:1-3.

2. Neogi T. Clinical practice: gout. N Engl J Med 2011;364:443-52.

3. Agudelo CA, Wise CM. Gout: diagnosis, pathogenesis, and clinical manifestations. Curr Opin Rheumatol 2001;13:234-9.

4. Chung TG, Kim HG, Song YS, Han SH, Kim JS, Kim HD, et al. Clinical manifestations of gout in Korea. Korean J Med 1997;53:84-92.

5. Peloquin LU, Graham JH. Gout of the patella; report of a case. N Engl J Med 1955;253:979-80.

6. Askin A, Inci R, Akan O, Demirdal S, Bayram K. A rare cause of knee pain in gout patients: tophi of patella. Arch Rheumatol 2015;30:71-4.

7. Clark S, Evans JM, Armstrong N, Schnitz W. Tophaceous gout with rare involvement of the patella. Radiol Case Rep 2016;11:380-5.

8. Ko KH, Hsu YC, Lee HS, Lee CH, Huang GS. Tophaceous gout of the knee: revisiting MRI patterns in 30 patients. J Clin Rheumatol 2010;16: 209-14.

9. Singh J, James SL, Kroon HM, Woertler K, Anderson SE, Jundt G, et al. Tumour and tumour-like lesions of the patella: a multicentre experience. Eur Radiol 2009;19:701-12. 